IGCS20_1295

\section{SURGICAL RESULTS OF ABDOMINAL VERSUS MINIMALLY INVASIVE RADICAL HYSTERECTOMY FOR CERVICAL CANCER IN SAN BORJA CLINICAL HOSPITAL SANTIAGO OF CHILE}

J Ibacache*, A Lagos, R Altamirano, O Nazzal, M Solari, E Pincheira, L Rojas, P Cervantes, E Suarez. Hospital San Borja Arriaran, Chile

\subsection{6/ijgc-2020-IGCS.243}

Upon the publication of the LACC study, increasing evidence has emerged regarding the superiority of open versus minimally invasive surgery (MIS) in the treatment of cervical cancer.

Introduction to report and compare the surgical results among woman undergoing surgery for cervical cancer with open versus MIS in San Borja Arriarán Hospital.

Methods retrospective analysis, involving patients with stage IA1 (lymphovascular invasion), IA2, or IB1 cervical cancer undergoing open or MIS between 2015 to 2019. Data obtained from oncology committee registry. Analysis of demographic, histological and surgical variables. Data analyzed using the Chi2, Student's t, and Fischer test with a 95\% confidence interval and a $\mathrm{P}$ value of $<0.05$.

Results 42 patients analyzed, 20 in the open surgery and 22 MIS group. The mean age $(46.2 \pm 2.9 \mathrm{v} / \mathrm{s} 46.9 \pm 2.37 ; \mathrm{P}$ $=0.49$ ). Surgical result, the number of nodes obtained in open surgery was significantly higher than in the MIS technique $(27.4 \pm 2.6 \mathrm{v} / \mathrm{s} 20.3 \pm 2.04 ; \mathrm{P}=0.038)$, with no differences in the number of positive nodes, compromise of surgical margins, parametrial compromise, tumor size or in lymphovascular invasion. There was no significant difference in adjuvance treatment. Due to the short follow-up period in the open surgery group, no recurrence or survival analysis was performed.

Conclusion Despite the small sample size, a significant difference in known variables of the oncological result, such as the number of lymph nodes obtained. This provides a first approach regarding oncological outcome. Survival analysis will be evaluated in future analysis.
IGCS20_1298

\section{OBESITY AND TYPE I ENDOMETRIAL CARCINOMA: AN OVERLY SIMPLIFIED MODEL?}

${ }^{1} \mathrm{C}$ Liao, ${ }^{2} \mathrm{M}$ Richardson* ${ }^{2} \mathrm{~K}$ Tran, ${ }^{3} \mathrm{C}$ Chan, ${ }^{3} \mathrm{AK}$ Mann, ${ }^{4} \mathrm{GL}$ Maxwell, ${ }^{5} \mathrm{CA}$ Hamilton, ${ }^{4} \mathrm{C}$ Tian, ${ }^{4} \mathrm{~K}$ Darcy, ${ }^{6} \mathrm{DS}$ Kapp, ${ }^{3} \mathrm{JK}$ Chan. ${ }^{1}$ Kaohsiung Veterans General Hospital, Taiwan; ${ }^{2}$ University of California, Los Angeles, USA; ${ }^{3}$ Palo Alto Medical Foundation, California Pacific Medical Center, Sutter Health, USA; ${ }^{4}$ Walter Reed National Military Medical Center, USA; ${ }^{5}$ Inova Schar Cancer Institute, USA; ${ }^{6}$ Stanford University School of Medicine, USA

\subsection{6/ijgc-2020-IGCS.244}

Objective To evaluate the relationship between obesity and incidence of type I endometrial cancer in two large population cohorts.

Methods From 2001 to 2016, incidence rates were estimated from United States Cancer Statistics data. Hysterectomy, pregnancy, and obesity rates were derived from the Behavioral Risk Factor Surveillance System (BRFSS). SEER*Stat and Joinpoint regression were used to calculate the incidence rate (per 100,000).

Results A total of 531,481 patients were diagnosed with endometrial cancer, and 385,821 were diagnosed with grade 1 or 2 endometrioid histology (82\% White, 6\% Black, 8\% Hispanic, 3\% Asian). Women aged 65-69 had the highest incidence (80.31). Incidence was highest in White women (20.0), followed by Hispanics (15.1), Blacks (12.8), and Asians (9.9).

The overall incidence of grade 1 and 2 endometrioid carcinoma decreased by $2 \%$ per year from 24.4 to 18.5 between 2001 and $2016(\mathrm{p}<0.001)$. Using survey data from BRFSS, from 2001 to 2016, the proportion of women with obesity increased from $21.1 \%$ to $30.2 \%$ (average annual increase of $2.3 \%, \mathrm{p}<0.001)$. Black women had the highest proportion of obesity (47.5\%), followed by Hispanics (33.7\%), Whites (28.1\%), and Asians (12.8\%). However, there was no increase in either grade 1 or 2 endometrioid carcinoma incidence over the study period, and no association with the increase in obesity rate.

Conclusion Older and White women are at higher risk of grade 1 or 2 endometrial cancer. Our combined analyses suggest that the decreasing incidence of type I endometrial cancer is not correlated with the rise in obesity.

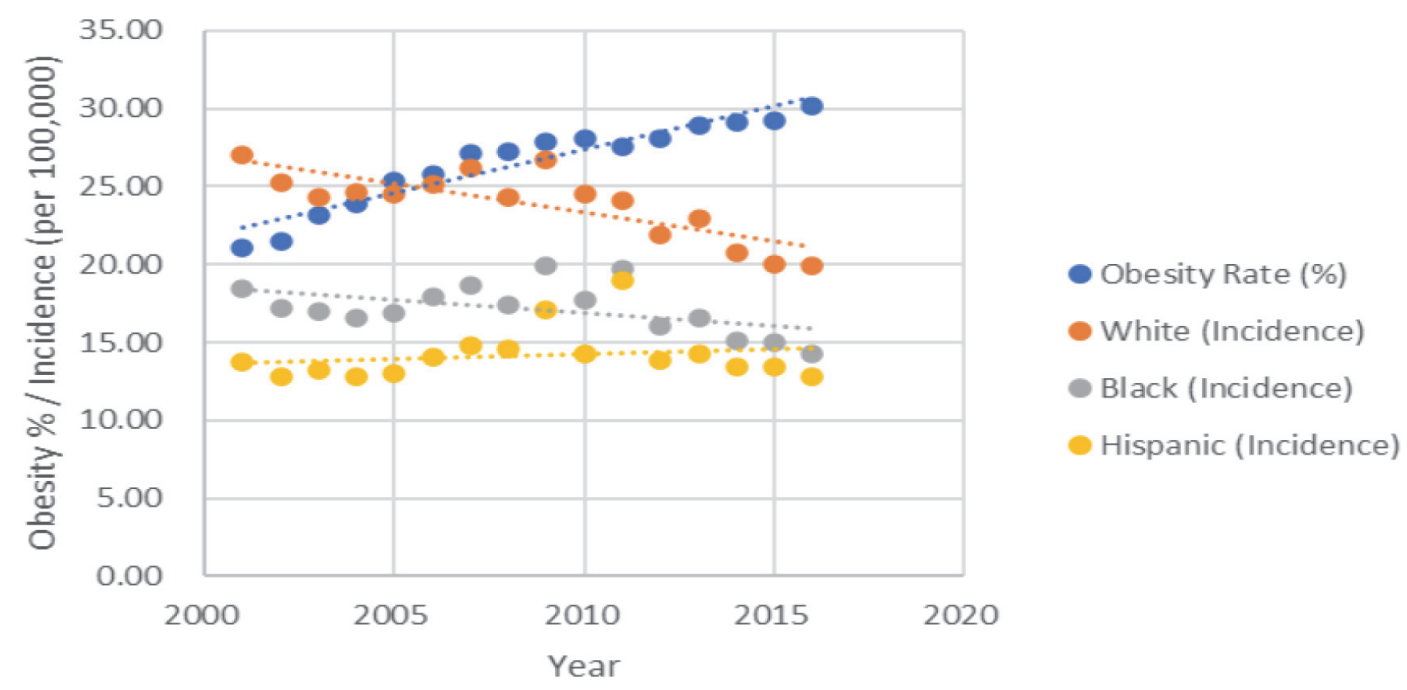

Abstract 284 Figure 1 